\title{
RESECCIÓN DE FRENILLO LINGUAL BAJO ANESTESIA GENERAL INHALATORIA: DESCRIPCIÓN DE TÉCNICA
}

\section{LINGUAL RESECTION OF THE BRAIN UNDER GENERAL INHALATION ANESTHESIA: DESCRIPTION OF THE TECHNIQUE}

\author{
Palacios-Vivar Diego $^{1 *}$, Varela-Ibañez Eduardo ${ }^{2}$, Calderón-Lumbreras Angélica ${ }^{3}$, Miranda-Villasana José ${ }^{4}$ \\ ${ }^{1}$ Cirujano Maxilofacial, Rotante en el servicio de Cirugía Craneofacial, Hospital DIF de Pachuca. México \\ ${ }^{2}$ Jefe del Servicio de Cirugia Craneofacial, Hospital Dif de Pachuca. México. \\ ${ }^{3}$ Medico Interno de pregrado, FacMed, Universidad Nacional Autonoma de México. México \\ ${ }^{4}$ Maestro en Educación, Jefe de servicio de Cirugía Maxilofacial del Hospital Regional "General Ignacio Zaragoza", \\ ISSSTE, Ciudad de México. México \\ *diegoepalaciosvivar@yahoo.com
}

\begin{abstract}
Resumen
El frenillo lingual es una banda de tejido fibroso o muscular recubierto por una membrana mucosa, su finalidad es ser un limitante para la función del órgano y permitir su desplazamiento dentro de sus márgenes fisiológicos. La anquiloglosia es una anormalidad congénita. Esta patología presenta un frenillo lingual corto o muy anterior; causando dificultad, limitación e imposibilidad de que la lengua cumpla su rol dentro del sistema estomatognatico. El tratamiento de resección del frenillo lingual o frenectomía se realiza por lo habitual con anestesia local. En ocasiones factores como problemas de conducta o una temprana edad del paciente hacen que se recurra a una anestesia en quirófano de tipo inhalatoria. Este tipo de anestesia brinda un estado de inconsciencia, analgesia por un corto plazo, posibilidad de trabajar con un paciente sin movimiento para procedimientos cortos. A continuación se presenta el procedimiento protocolario de frenectomía de pacientes menores a 4 años realizado bajo anestesia general inhalatoria en el Centro Craneofacial del Hospital DIF del Niño de Pachuca en el periodo del $2012-2014$.
\end{abstract}

Palabras clave: Frenillo lingual corto, anquiloglosia, frenectomía, anestesia general inhalatoria, sevoluorano, óxido nitroso.

\begin{abstract}
Ankyloglossia is a common congenital abnormality in which the lingual frenum attaches tongue to floor of mouth and sometimes alveolar ridge, is short or anterior; causing difficulty and limitation in movement. Treatment resection or frenectomy, includes as every procedure anesthesia, which comes in various forms, for this type of procedure and patient age anesthesia inhalatoria with sevoflurane as first option. Treating or frenulum resection is performed frenectomy usual with local anesthesia. Factors such as behavioral problems or age of the patient make recourse to an inhalational anesthesia in operating room type. This kind of anesthesia provides a state of unconsciousness, analgesia for a short-term possibility of working with a patient without moving for short procedures. In this paper we present frenectomy procedure for patients under 4 years performed under general inhalatory anesthesia at the Craniofacial Center in the Hospital del Niño DIF of Pachuca in the period form 2012 to 2014
\end{abstract}

Key words: Tongue tie, ankyloglossia, frenectomy, general inhalatory anesthesia, sevoflurane, nitrous oxide.

\section{INTRODUCCIÓN}

El frenillo corto se conoce como anquiloglosia, siendo esta un remanente embriológico de la superficie ventral de la lengua al piso de la boca. Este término de la raíz ankylos que significa curvatura, anular, disminuir o suprimir y del sufijo glossia que indica lengua., ${ }^{1,2}$ La prevalencia varia según el autor desde $0.5 \%$ al $4 \%$ de la población. ${ }^{1}$ El diagnóstico inicial se establece al observar la imposibilidad de la lengua de contactar con el paladar duro con la boca abierta, incapaz de protruir mas allá de 1 a $2 \mathrm{~cm}$ por fuera de incisivos inferiores. La anquiloglosia en muchos casos es heredada. ${ }^{3,4}$ 
La limitante de este órgano muscular genera lo siguientes problemas. $^{5,6}$

1) Dificultad en la succión, inflamación en el pezón de la madre.

2) Dificultad para la deglución, interposición lengua entre los incisivos ocasionando una deglución atípica.

3) Alteraciones en la fonación: Consonantes linguodentolabiales. Sustituyendo el sonido "f" "v" por "fh".

4) Problemas ortopédicos / ortopédico: Diastema de incisivos inferiores por inserción a nivel de cresta alveolar, desarrollo deficiente del paladar y mordida abierta.

No hay una etiología particular que ocasione la anquiloglosia, pero se han relacionado con los Síndromes Orofaciodigital, Síndrome Opitz, Síndrome Simpson-GolabiBehmel, Paladar Hendido asociado al cromosoma X, Síndrome de Beckwith-Wiedemann y enfermedad de Riga-fede. $\mathrm{Su}$ fisiopatología es referida por Naimer y col a un desorden en la falta de diferenciación tisular durante el período embrionario. ${ }^{8,9}$ La valoración clínica es indispensable para el diagnóstico (Figura 1 y 2). Se recomienda iniciar con una fase de observación del recién nacido, y problemas asociados a lactancia materna, y posteriormente problemas de dicción, autolisis, alteraciones en posición de incisivos inferiores. Existen varias clasificaciónes, que tienen como objetivo determinar la gravedad de esta alteración lingual, y si es necesario realizar un tratamiento quirúrgico. ${ }^{1,6,10}$ Para categorizar el nivel de inserción y su severidad se toman en cuenta tres clasificaciones.

1) Coryllos: Elasticidad del frenillo y posición de la punta lingual con respecto a estructuras anatómicas. Tipo 1: Frenillo delgado y elástico; se adhiere de la punta de la lengua al proceso alveolar de modo que la lengua adquiera una forma de corazón. Tipo 2: Frenillo delgado y elástico; 2 a 4mm detrás de la punta lingual, se adhiere al proceso alveolar. Tipo 3: Grueso y fibroso, frenillo no elástico, se adhiere de la mitad de la lengua al piso de boca. Tipo 4: El frenillo no se observa pero se siente, con adherencia fibrosa, submucosa, y brillante adherencia, de la base la lengua al piso de la boca.

2) Hazzelbaker: Valores numéricos del 0 al 3 para valorar apariencia y función. (Tabla I y II)

3) Kotlow: medición de longitud de lengua desde la inserción del frenillo hasta la punta (Tabla III)

El principio del tratamiento consiste en realizar una remoción quirúrgica vertical del frenillo acompañado con frecuencia de disección de haces superficiales del musculo geniogloso. La terapéutica se complementa con fisioterapia y terapia de lenguaje. Existen diversos procedimientos descritos los cuales varían de acuerdo a la edad del paciente, grado de anquilosis, recursos del centro de atención y preferencia del cirujano. El tratamiento quirúrgico se puede realizar bajo anestesia local infiltrativa, sedación consciente, anestesia general bajo intubación o inhalatoria; siendo este último el

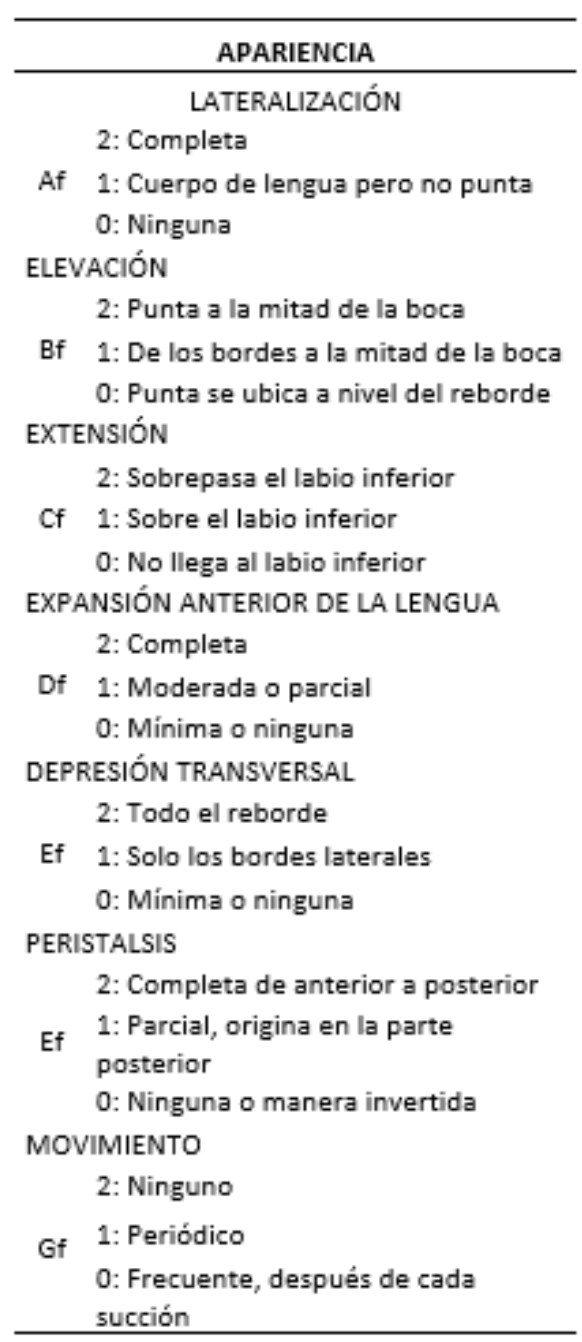

Tabla 1. Análisis De Hazelbaker

utilizado en nuestro centro medico para pacientes menores a 4 años. ${ }^{5,11}$ Es de consenso que el manejo de la ansiedad, incomodidad y cooperación de paciente pediátrico es una de las tareas más difíciles de la cirugía oral en la consultorio dental. En casos que el padecimiento o condiciones propias del paciente no permitan un proceso ambulatorio es necesario continuar con un tratamiento bajo anestesia general el cual nos provee de reducción de la ansiedad, inmovilidad, comodidad, amnesia, alcanzar analgesia y asegurar estabilidad biodinámica. ${ }^{12,13}$

A continuación presentamos un breve resumen de los agentes inhalatorios o gases anestésicos utilizados para inducir y mantener la anestesia general, recordando que todos los inhaladores deprimen potencialmente el sistema ventilatorio por lo cual el manejo de estos medicamentos debe ser por parte de personal entrenado en anestesiología. ${ }^{14}$ Óxido nitroso: 


\begin{tabular}{|c|c|}
\hline \multicolumn{2}{|c|}{ FUNCIÓN } \\
\hline \multicolumn{2}{|c|}{ APARIENCIA DE LENGUA AL LEVANTARLA } \\
\hline & 2: Redonda o cuadrada \\
\hline \multirow[t]{2}{*}{ Ap } & 1: Signo de hendidura en la punta \\
\hline & 0: V o de Corazón \\
\hline \multicolumn{2}{|c|}{ ELASTICIDAD DEL FRENILLO } \\
\hline & 2: Muy elástica \\
\hline \multirow[t]{2}{*}{ Bp } & 1: Moderadamente elástica \\
\hline & 0: Poca o ninguna elasticidad \\
\hline \multicolumn{2}{|c|}{ LOGNITUD DEL FRENILLO } \\
\hline & $2:>5 \mathrm{~cm}$ \\
\hline \multirow[t]{2}{*}{ Cp } & $1: 1 \mathrm{~cm}$ \\
\hline & $0:<1 \mathrm{Cm}$ \\
\hline \multicolumn{2}{|c|}{ INSERCIÓN DE FRENILLOS EN LENGUA } \\
\hline & 2: En la base \\
\hline \multirow[t]{2}{*}{ Dp } & 1: En la punta \\
\hline & $0:$ No se percibe \\
\hline \multicolumn{2}{|c|}{ INSERCIÓN DEL FRENILLO AL PROCESO ALVEOLAR } \\
\hline & 2: Inserción en el piso de boca \\
\hline \multirow[t]{2}{*}{ Ep } & 1: Inserción debajo del reborde \\
\hline & 0: Inserción en el reborde \\
\hline
\end{tabular}

Tabla 2. Análisis De Hazelbaker

\begin{tabular}{lll}
\hline CLASE 0 & Mayor a $16 \mathrm{~mm}$ & Aceptable \\
CLASE I & 12 a $15 \mathrm{~mm}$ & Leve \\
CLASE II & 8 a $11 \mathrm{~mm}$ & Moderada \\
CLASE III & 3 a $7 \mathrm{~mm}$ & Severa \\
CLAVE IV & Menor de $3 \mathrm{~mm}$ & Completa \\
\hline
\end{tabular}

Tabla 3. Valores De Kotlow

Es ansiolítico, analgésico, amnésico, y efectos de sedación. No es un potente anestésico, pero posee un amplio margen de seguridad, y pocos efectos residuales. ${ }^{14,15} \mathrm{Su}$ baja solubilidad que permite un equilibrio entre el alveolo pulmonar, sangre, y cerebro. La desventaja es que por si solo no es capaz de inducir a la anestesia general. ${ }^{16,17}$ Sevofluorano (riflourmetiletel): Gas anestésico, popular en Japón por varios años, recientemente incorporado en el uso rutinario en México y Latinoamérica. Tiene la potencia suficiente para inducir a la anestesia general. Parámetros aceptables se pueden mantener durante su uso. A diferencia del halotano el sevofluorano produce menor depresión miocárdica, arritmia, riesgo disminuido de hipotensión y bradicardia. Lo que incluye un límite

\begin{tabular}{ccc}
\hline & Número & Porcentaje \\
\hline SEXO & 68 & $100 \%$ \\
Masculino & 49 & $72,10 \%$ \\
Femenino & 19 & $27.9 \%$ \\
Promedio de edad & 2 años 3 meses \\
\hline
\end{tabular}

Tabla 4. Pacientes periodo 2012-2014 Hospital Pediátrico DIF de Pachuca

de $1.0 \mathrm{ug} / \mathrm{kg}$ de epinefrina en combinación con halotano. Los agentes tienen baja solubilidad en el torrente sanguíneo, rápido para la inducción. Con un nivel sanguíneo del Oxido nitroso de 0.47 , sevofluorano 0.47 , halotano 2.3 . La recuperación de la anestesia inhalatoria depende del tiempo de duración. Estudios clínicos han determinado que la duración de 4 minutos, se presenta con una apertura ocular de 102 segundos con halotano, y 167 segundos con sevofluorano. ${ }^{14,17}$

\section{PROCEDIMIENTO}

Se coloca monitoreo cardiaco, óxímetro, placa de electrocauterio, se realiza la administración de anestesia general inhalatoria. Inicia procedimiento mediante anestésico tópico en spray de lidocaína, con tracción mediante la sonda de Petit, el frenillo se eleva a través de la hendidura, se identifica las carunculares sublinguales y submandibulares, y se realiza corte con electrobisturí protegido a nivel del frenillo para limitar el campo de acción y generar un corte preciso. Se corrobora la distensión de lengua, hemostasia de la herida y se termina acto quirúrgico. Se coloca una cánula oral para evitar retro posición lingual, se verifica la ventilación, saturación adecuada y se traslada paciente a recuperación bajo supervisión y un monitor con óxímetro hasta que el paciente este despierto. (Figura 3,4,5,6)

\section{RESULTADOS ESPERADOS}

Un procedimiento de resección de frenillo lingual exitoso debe lograr que la punta de la lengua en apertura bucal toque. Protruir fuera de la boca sin presentar características de bífida, extenderse sobre los labios sin fuerza excesiva sobre dientes antero inferiores, generar una adecuada deglución y en pacientes lactantes no presentar dificultad o dolor para la madre al momento de la succión. En el servicio de la Clínica de Craneofacial del Hospital DIF del niño Pachuca, se han tratado en un periodo de 3 años, 68 paciente, con un promedio edad 3años 3 meses, con el menor de 6 meses y mayor de año 6 años 2 meses. Recordando que al ser diagnosticados en 


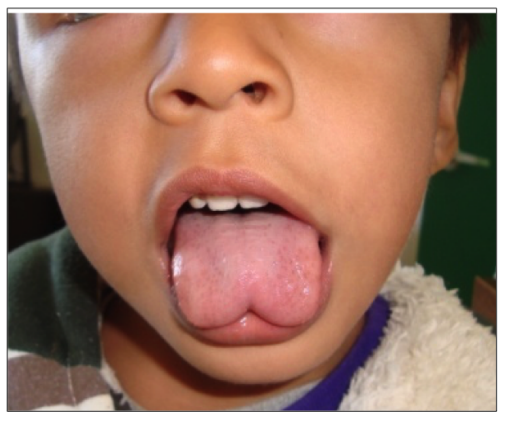

Fig. 1. Lengua en forma de corazón

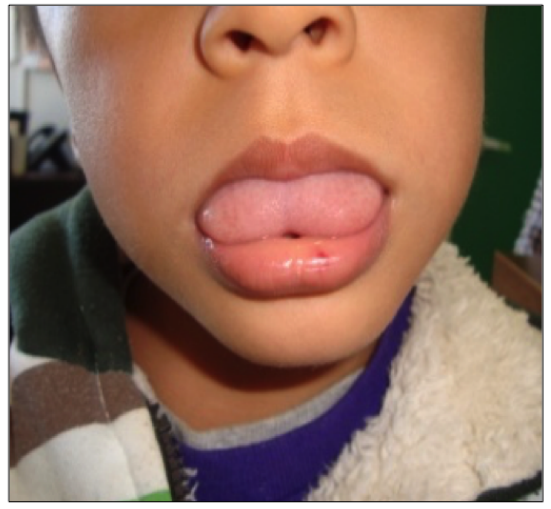

Fig. 2. Limitación en la protrusión lingual

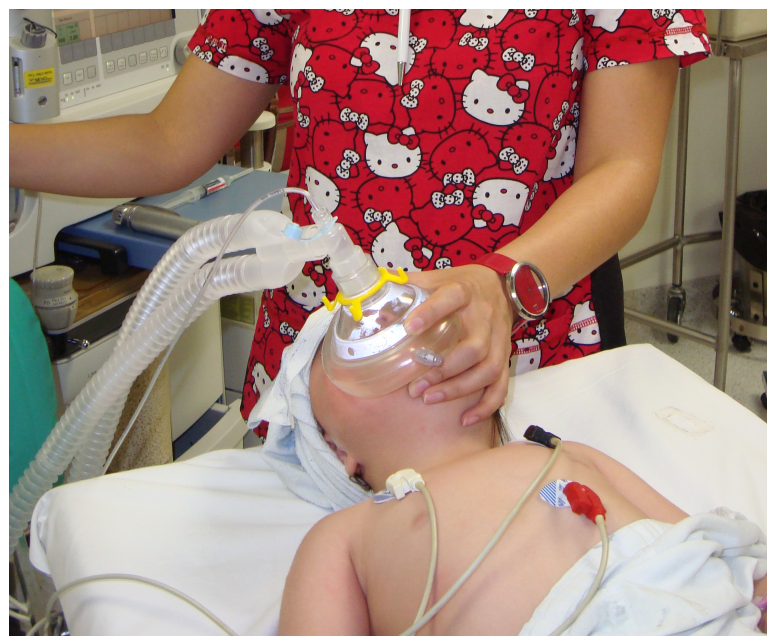

Fig. 3. Inducción anestesia inhalatoria

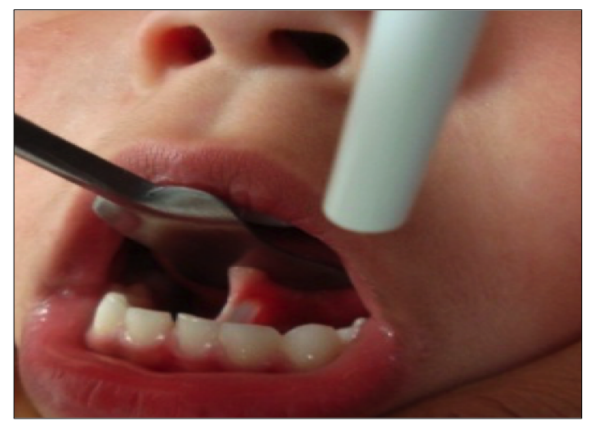

Fig. 4. Tracción de frenillo con cánula de Pettit

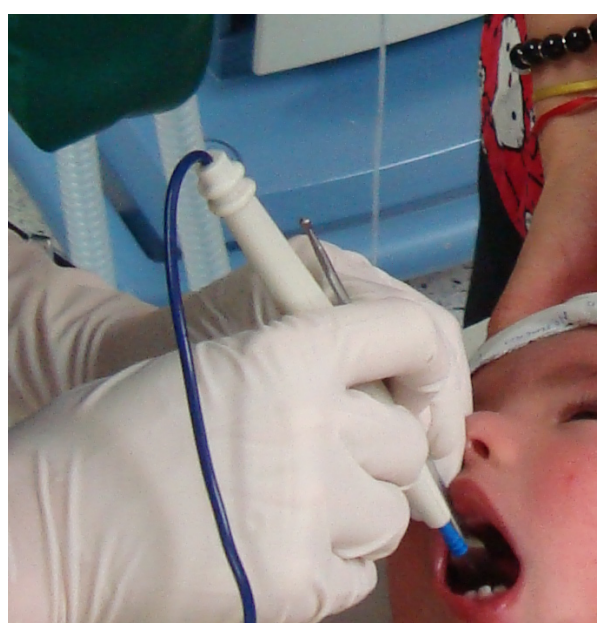

Fig. 5. Resección con Electrobisturí

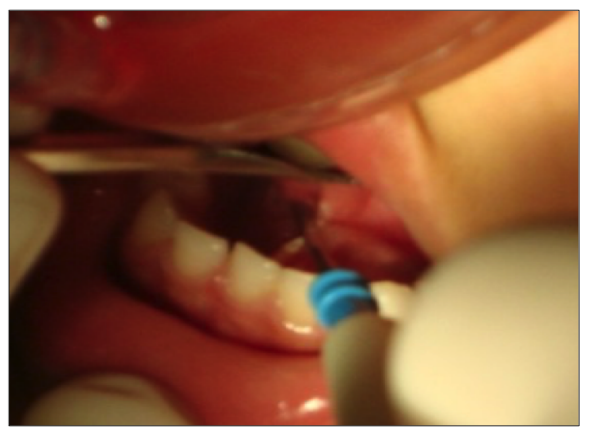

Fig. 6. Herida en frenillo lingual 
menores de 4 meses, se puede realizar el procedimiento con anestesia local debido a la poca inervación del frenillo a edad temprana y la facilidad de inmovilizar al paciente.

\section{DISCUSIÓN}

La anquiloglosia es un trastorno del desarrollo frecuente, identificado por dificultad de succión del infante al pecho de la madre, o dificultad para articular los fonemas ya mencionados. ${ }^{4,7}$ El procedimiento de resección se debe realizar en una etapa temprana, no se debe posponer por argumentos como falta de colaboración o espera que el involucione el problema con el desarrollo del individuo. ${ }^{2,10}$ Gooden comenta que deben tomarse en cuenta los riesgos aumentados que genera el uso de agendes inductores. $\mathrm{Su}$ potencial capacidad de desencadenar una reacción alérgica o una depresión del sistema nervioso profunda si no se usa dosis adecuadas o el paciente es más sensibles por alteración propia de su fisiología. ${ }^{15} \mathrm{Es}$ indispensable realizar este procedimiento manejado por un especialista en anestesiología con experiencia en el manejo de pacientes pediátricos o mejor aún con subespecialista anestesiólogo pediatra. ${ }^{6,7,11}$

La profundidad de la resección dependerá si es una anquilosis mucosa y/o muscular para obtener una liberación que otorgue a la lengua una adecuada tracción y movimiento. ${ }^{2,10}$ Regal refiere que el tratamiento debe ser realizado a una edad temprana para evitar trastornos en la succión y anomalías de lenguaje no neurológica de base. ${ }^{?}$ No se debería esperar que el infante crezca, y sea colaborador. ${ }^{5}$ Las complicaciones locales son poco frecuentes, generalmente una ligera tumefacción, equimosis en piso de boca y dolor leve. ${ }^{3}$ La anestesia general inhalatoria tiene indicaciones diferentes a la anestesia general mediante intubación oro/naso traqueal. El tiempo de acción de la primera es de pocos minutos como máximo de 20 minutos; no están indicados procedimientos que generen sangrado o presencia de irrigación porque la vía aérea inferior no se encuentra protegida. ${ }^{7,14,15}$ En la anestesia con intubación se necesita obtener acceder vía venosa permeable, administrar una serie numerosa de fármacos para inducir a una anestesia general inducir e instalar una sonda traqueal. Aumento el riesgo por contacto a varios fármacos, dolor por la colocación de la sonda y un mayor tiempo de recuperación. ${ }^{14,15}$

Con el paciente bajo anestesia inhalatoria el procedimiento quirúrgico es sencillo, rápido realizarlo. El uso con electobisturí no genera sangrado, y además los bordes de la herida no ameritan afrontamiento con sutura. El hecho de ser un procedimiento en teoría mecánicamente sencillo, amerita ser realizado por un profesional que conozca bien la anatomía local para 10 reducir el riesgo de daño a estructuras anexas al frenillo.

\section{CONCLUSIONES}

1) Un instrumento de medida es un procedimiento estandarizado que permite obtener un conocimiento objetivo de una persona, producto, sistema o institución.

2) Las inferencias que se extraigan de las puntuaciones de un instrumento de medida son siempre para un uso, contexto y población determinada.

3) Tener instrumentos de evaluación adecuados no garantiza un proceso evaluativo exitoso.

4) Se puede elaborar un manual del instrumento que permita su utilización a otras personas o instituciones interesadas. Este debe recoger con todo detalle las características relevantes.

5) Si un instrumento es válido tiene significancia social, y asume una función tanto científica como política (estrategias).

Conflicto de intereses y financiamiento Los autores declaran no tener conflicto de intereses, haber cumplido con los requisitos de autoría y haber autofinanciado este artículo.

\section{Referencias}

1 Gonzales D, Costa M. Prevalence of ankylogossia in newborns in Asturias. Asociación española de pediatría 2013; 1 : 341-346

2 Baldani MH, Lopes MDL, Scheidt W. A Prevalencia de las alteraciones bucales atentidos en la clínica de bebes de Ponta Grossa - PR, Brasil. Pesqui Odontol Bras 2001; 15(4): 302-307.

3 Kupietzky A, Botzer E. Ankyloglossia in the infant and young Child: Clinical suggestion for diagnosis and management. Pediatr Dent 2005; 27(1): 40-4

4 Guillermo B. Anquilogia: revisión de la literatura y reporte de un caso. revista estomatológica 2005; 13 (2)..

5 Pérez N, López M. Anquiloglosia en niños de 5-11 años de edad. Diagnóstico y tratamiento. Rev Cubana Estomatología. 39(3): 282-301.

6 Nahás Pires Correa, Maria salete, y col. Anquiloglosia: ¿Cuándo intervenir? Revisión y reporte de caso; Acta Odontológica Venezolana 2009; 47(3).

7 O'Callahan C, Macary S, Clemente S. The effects of office-based frenotomy for anterior and posterior ankyloglossia on breast- feeding. Int J Pediatr Otorhinolaryngol. 2013;77:827-32.

8 Mintz S, Siegel M, Seider P. An overview of oral frena and their association with multiple syndromic and nonsyndromic conditions. Oral Surg Oral Med Oral Phatol Oral Radiol Endod 2005; 99(3): 321-4.

9 Segal L, Stephenson R, Dawes M, Feldman P. Prevalence, diagnosis, and treatment of ankyloglossia: methodologic review. Can Fam Physician 2007; 53(6): 1027-1033.

10 Cinar F, Onat N. Prevalence and consequences of a forgotten entity: ankyloglossia. Plast Reconstr Surg 2005; 115(1): 355-356. 
11 Rosas G, González M, González B. Anquiloglosia parcial (incompleta): Reporte de un caso y revisión de la literatura. Revista ADM 2009; 65 (2): 42-47

12 Klockars T, Pitkaranta A. Inheritance of ankyloglossia (tongue- tie). Clin Genet 2009; 75: 98-99.

13 Hossam Z, Ayman A. Safety and consumption of sevoflurane versus desflurane using target controlled anesthesia in children. Egyptian Journal of Anaesthesia 2014;30:325-329

14 Gooden R, Tennant I, James I. The incidence of emergence delirium and risk factors following sevoflurane use in pediatric patients for day case surgery. Brazilian Journal of Anesthesiology 2014; 64(6): 413-418.

15 Hasanein R, El-Sayed W. The effect of nebulized lidocaine hydrochloride on emergence from sevoflurane anesthesia in children undergoing Tosillectomy, Egyptian Journal of Anaesthesia 2013; 29(4): 351-356.

16 Braz M, Braz L, Barbosa B, y cols. DNA damage in patients who underwent minimally invasive surgery under inhalation or intravenous anesthesia. Mutation Research 2011; 726: 251-254

17 Regal N. Dislalias. Rev Cubana Ort. 14(2): 89-93. .

Recibido: 19 de Junio de 2016

Aceptado: 24 de Julio de 2016 